\title{
Stress prevention by modulation of autonomic nervous system (heart rate variability): A preliminary study using transcranial direct current stimulation
}

\author{
Eduardo Manuel Gonçalves ${ }^{1,2 *}$, Saul Neves de Jesus ${ }^{2,3}$ \\ ${ }^{1}$ Department of Psychiatry, Mental Health of Hospital of Faro, Faro, Portugal \\ ${ }^{2}$ Department of Psychology (Health Psychology), Faculty of Social Sciences, University of Algarve, Faro, Portugal \\ ${ }^{3} \mathrm{CIEO}$ - Research Center for Spatial and Organizational Dynamics, University of Algarve, Faro, Portugal \\ Email: *eduar.goncalves@gmail.com
}

Received 11 February 2012; revised 10 March 2012; accepted 31 March 2012

\begin{abstract}
Introduction: Transcranial Direct Current Stimulation (tDCS) is a non-invasive, technique for brain stimulation. Anodal stimulation causes neuronal depolarisation and long-term potentiation, while cathodal stimulation causes hyperpolarisation and longterm depression. Stressors are associated with an increase in sympathetic cardiac control, a decrease in parasympathetic control, or both. Associated with these reactions is a frequently reported increase in Low Frequency (LF) Heart Rate Variability (HRV), a decrease in High Frequency (HF) power, and/or an increase in the LF/HF ratio. Objectives and Aims: The present work aims to explore the tDCS potential in the modulation of the Autonomic Nervous System (ANS), through indirect stimulation of Anterior Cingulate Cortex (ACC). Methods: Two subjects, a 39 year old female and a 49 year old male, gave informed consent. Saline soaked synthetic sponges involving two, thick, metalic (stainless steel) rectangles, with an area of $25 \mathrm{~cm}^{2}$ each have been used as electrodes, connected to Iomed Phoresor II Auto device. It has been delivered a $\mathbf{2} \mathrm{mA}$ current, for $\mathbf{2 0}$ minutes, over the left Dorsolateral Prefrontal Cortex (DLPFC) (Anode). Spectrum analysis (cStress software) of HRV has been performed before and after tDCS administration. Results: The female/male subject results of $L F$ power, $H F$ power and $L F / H F$ ratio, before tDCS administration, were, respectively: $50.1 \mathrm{nu} / 60$ $\mathrm{nu}, 46.1 \mathrm{nu} / 21.7 \mathrm{nu}$ and 1.087/2.771; and, after tDCS administration, respectively: $33.5 \mathrm{nu} / 52.7 \mathrm{nu}, 47.6 \mathrm{nu} /$ $22.8 \mathrm{nu}$ and $0.704 / 2.312$. Conclusions: tDCS over the left DLPFC (left ACC) increased parasympathetic activity and decreased sympathetic activity, suggesting the importance of tDCS in the management of stress-related disorders.
\end{abstract}

"Corresponding author.
Keywords: Transcranial Direct Current Stimulation; Left Dorsolateral Prefrontal Cortex; Left Anterior Cingulate Cortex; Autonomic Nervous System; Heart Rate Variability; Stress

\section{INTRODUCTION}

\subsection{Definition and Applications of Transcranial Direct Current Stimulation}

Transcranial Direct Current Stimulation (tDCS) is a simple, non-invasive, technique for brain stimulation that induces prolonged functional changes in the cerebral cortex [1]; essentially it consists in delivering for a few minutes a weak direct current (below the perceptual threshold) over the scalp: the resulting constant electric field penetrates the skull and influences neuronal function. The electrical field of tDCS penetrating into the scalp induces changes in brain excitability. Stimulation of different cortical areas had resulted in modifications of perceptual, cognitive, and behavioral functions, and it can induce beneficial effects in brain disorders [2-27] (Figure 1). The tDCS is a form of neurostimulation that may be used to safely treat a variety of clinical conditions including depression, obsessive-compulsive disorder, Tourette's disease, Alzheimer's disease, migraine, and central and neuropathic chronic pain. It can also relieve the symptoms of narcotic withdrawal and reduce cravings for drugs, including nicotine and alcohol. It can be used to increase frontal lobe functioning and reduce impulsivity and distractibility in persons with attention deficit disorder; tDCS also boosts verbal and motor skills and improve learning and memory, in healthy people. Initial studies in humans aimed at treating or modifying psychiatric diseases, particularly depression. Anodal stimulation diminish depressive symptoms [28], while cathodal stimulation reduce manic symptoms [29]. The basic treatment consists of a series of five 20-minute 


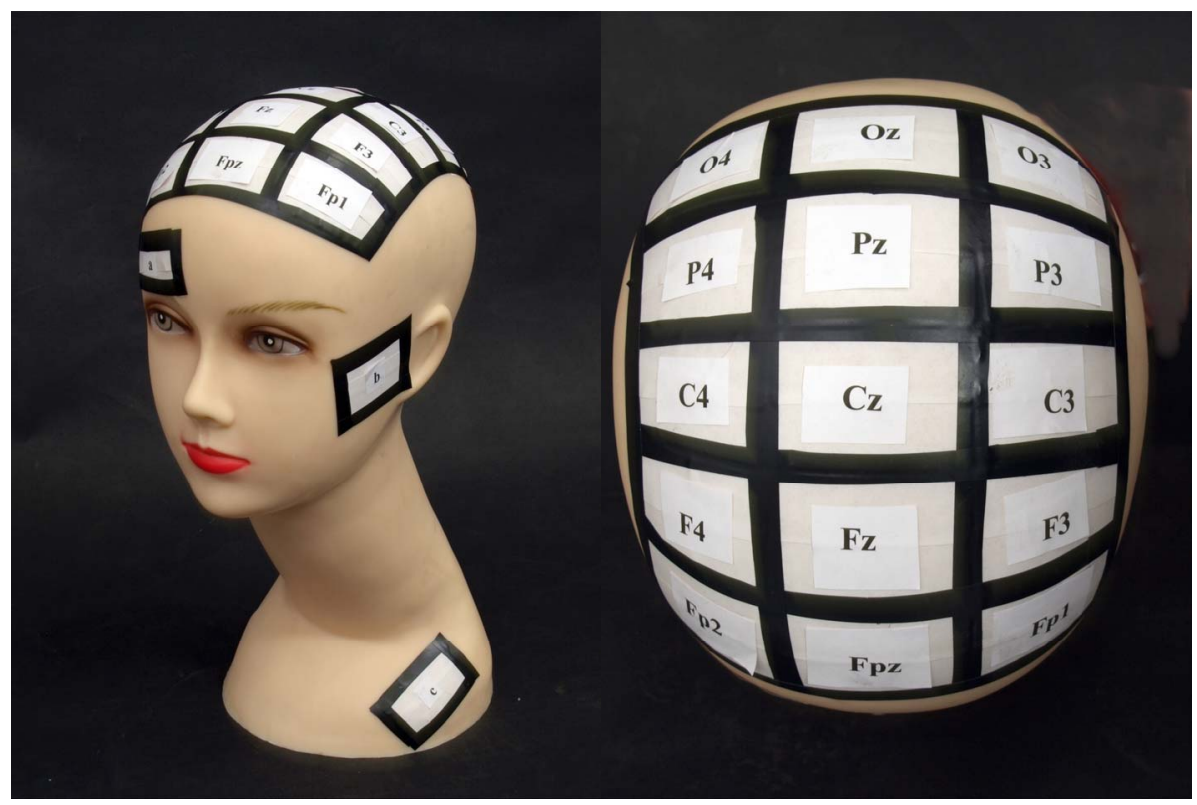

\begin{tabular}{ccl}
\hline Anode & Cathode & \multicolumn{1}{c}{ Effects } \\
\hline O4 + Fp2 & c & Visual Acuity \\
F4 & b & Declarative Memory \\
Pz & a & Spatial Acuity \\
P3 & C3 & Picture Naming \\
P3 & a & Language Learning, Precision of Gestures \\
C3 + Fp1 & a & Working Memory, Sleep Quality, Pain Relief \\
F3 & F4 & Decision Making, Mathematics Competence \\
F3 & a & Verbal Fluency \\
Fp1 & Fp2 & Alcohol Craving \\
Fp1 & a & Assumption of Risky Behaviors, Planification Ability, Depression \\
\end{tabular}

Figure 1. Schematic drawing of electrode positions suited for tDCS from Prefrontal Cortex (Fp2, Fpz, Fp1) to Occipital Cortex $(\mathrm{O} 4, \mathrm{Oz}, \mathrm{O} 3)$, and expectable stimulatory effects. The cathode is positioned such that the resulting current flow from the cathode to the anode allows an effective modulation of neuronal excitability under the anode [Reference electrode - the cathode in these examples, including a (right supra-orbital area), b (left zygomatic arch), c (left shoulder) - does not mean necessarily that this electrode is functionally inert, but that neural excitability changes under this electrode are beyond the scope of interest with regard to a specific experimental setting] (adapted from M. A. Nistche et al., Transcranial Direct Current Stimulation: State of the Art 2008, Brain Stimulation Elsevier, Inc.).

sessions over five consecutive days. While the patient is seated, two $5 \mathrm{~cm} \times 5 \mathrm{~cm}$ non-metallic conductive rubber electrodes are placed on selected locations of the scalp and covered by saline soaked sponges and held in place by elastic headbands. After the electrodes are properly placed, a tDCS device powered by a 9-volt battery is used to send a steady electrical current of $1-2$ miliAmpère (mA). Fregni et al. (2006) [27] reported on a randomized, sham-controlled, clinical trial of tDCS in the treatment of 10 patients diagnosed with major depression. The level of depression was evaluated before and after treatment by means of the Hamilton Depression Rating Scale (HDRS) and Beck Depression Inventory (BDI). Patients were randomly assigned to one of two groups, an active treatment group that received $1 \mathrm{~mA}$ anodal (electrode positively charged) tDCS over the Left Dorsolateral Prefrontal Cortex (DLPFC) and cathodal (electrode negatively charged) stimulation over the contralateral supraorbital area (just above right eyebrow) versus a sham treatment group that received the identical treatment but with the tDCS device turned off. Both groups received 20 minutes of actual or sham stimulation once a 
day for five consecutive days. The active treatment group showed a significantly greater reduction in depression scores on the post-treatment HDRS and BDI as compared to the sham treatment group $(70 \%$ versus $30 \%$, respectively).

\subsection{Mechanism of Action of Transcranial Direct Current Stimulation}

Systematic animal studies in anesthetized rats demonstrated that weak direct currents, delivered by intracerebral or epidural electrodes induce cortical activity and excitability diminutions or enhancements, which can be stable long after the end of stimulation [30]. Stimulation with the negative pole (cathode) placed over a selected cortical region will decrease neuronal activity under the electrode whereas stimulation with the positive pole (anode) will increase neuronal activity under the electrode. Changes in spontaneous neuronal firing rates, coupled with synaptic neuroplasticity, contribute to intraand post-stimulation effects respectively. The changes are long-lasting -5 to 10 minutes of continuous stimulation produced effects up to 5 hours afterwards. Anodal stimulation shifts neuronal resting membrane potential towards depolarisation, while cathodal stimulation shifts resting membrane potential towards hyperpolarisation. tDCS induces changes by altering the strength of synaptic transmission. Anodal toes could induce Long-Term Potentiation (LTP), through increased pre-synaptic activity coupled with postsynaptic depolarisation; conversely, cathodal toes could induce Long-Term Depression (LTD), through reduced presynaptic discharge and postsynaptic hyperpolarisation. The evidence for a neuroplastic synaptic contribution to the effects of tDCS is thus compeling. Respecting the physical parameters and practical application of tDCS, the efficacy of tDCS to induce acute modifications of membrane polarity depends on current density, which determines the induced electrical field strength [31], and is the quotient of current strength and electrode size. Larger current densities result in stronger effects of tDCS [32,33]. Another important parameter of tDCS is stimulation duration. With constant current density, increasing stimulation duration determine the occurrence and duration of after-effects in humans and animals [30,32,34,35]; direct currents are generally delivered via a pair of sponge electrodes moistened with tap water or $\mathrm{NaCl}$ solution (size between 25 and $35 \mathrm{~cm}^{2}$ in different studies) $[12,32,33,36,37]$. Increasing focality of tDCS may be achieved by: 1) reducing electrode size, but keeping current density constant, for the electrode that is intended to affect the underlying cortex; 2) increasing the size, and thus reducing current density, of the electrode, which should not affect the underlying cortex; or 3) using an extra-encephalic reference. Regarding the time course of tDCS-induced modulations of cortical excitability, because increasing current density will increase cutaneous pain sensation and might affect different populations of neurons (because the larger the current density, the greater the depth penetration of the effective electrical field), it is suggested to increase stimulation duration and not current density, if a prolongation of the effects of tDCS for an extended time course is wanted $[32,34,35]$. When applied for several minutes, tDCS produces lasting effects in the human motor cortex. These are stable for up to about an hour if tDCS is applied for 9 - 13 minutes [32,34,35,38].

\subsection{Safety Procedures of Transcranial Direct Current Stimulation}

In relation with safety procedures, generation of electrochemically produced toxins and electrode dissolution products at the electrode-tissue interface are only risks of tDCS for the skin contact, because there is no brainelectrode interface. Subjects should be specifically interviewed for the existence of skin diseases (also in the past) and the condition of the skin under the electrodes should be inspected before and after tDCS. Conventional electrical brain stimulation can cause excitotoxic damage to overdriven neurons [39]. This is not applicable to tDCS. The stimulation device should guarantee a constant current strength, because current strength determines the intensity of the electrical field in tissue and a constant voltage device could result in unwanted increases in current strength, if resistance decreases. Stimulation durations, which are likely to result in excitability changes lasting more than one hour, should be applied with caution, because changes lasting that long could be consolidated and stabilized, leading to unintended or adverse effects. The same applies for repeated application of tDCS to the same brain region without an appropriate interval between sessions. Subjects should be free of any illness that may increase the risk of stimulation, for example, neurologic diseases such as epilepsy or acute eczema under the electrodes. Furthermore, they should have no metallic implants near the electrodes. Seizures do not appear to be a risk for healthy subjects; however, this may not be true for patients with epilepsy [40].

\section{MODULATION OF AUTONOMIC NERVOUS SYSTEM (HEART RATE VARIABILITY) BY TRANSCRANIAL DIRECT CURRENT STIMULATION: A PILOT STUDY PRELIMINARY RESULTS WITH IMPORTANCE TO RESILIENCE TO STRESS SCIENCE}

\subsection{Heart Rate Variability}

The heart is a pump, though a complex one in structure and regulation, with two sides - a left side and right side 
pump - that work in concert, each with an antechamber (the atrium) and main pumping chamber (the ventricle), and valves that ensure a single direction of blood flow, from the body into the atria, then to the ventricles, and then back out to the body. The higher pumping pressure of the heart's left side matches the wider distribution of blood associated with the array of organs and tissues dependent on it for proper perfusion. The right side of the heart receives deoxygenated blood from body tissues and organs through the venous return system. The right side of the heart directs this blood to the lungs, where carbon dioxide and associated metabolic by-products are exchanged for oxygen. The rate, force, and timing of myocardial contraction are determined by self-regulatory elements, direct inputs from the autonomic nervous system, and ancillary circulatory elements, which together determine Cardiac Output (CO), the volume of blood in liters ejected by the left ventricle per unit of time (Figure 2). Self-regulatory elements include the very nature of myocardial cells, which are unique in that they are excited by the spread of excitation from contiguous cells. The heart also includes two key electrophysiological systems: the Sino-Atrial (SA) node or cardiac pacemaker, which is located in the right atrial wall, and spontaneously originates successive waves of excitation that then proliferate throughout the cardiac chambers; and the Atrio-Ventricular (AV) node, which originates in the atrial septum, projects down to the ventricular septum, and has two branches that spread over the endocardial surfaces of the ventricles. The Autonomic Nervous System (ANS), working directly and through the circulation, plays a key role in the regulation of cardiac function. The regulation of AV node "firing" is influenced by nerve endings associated with the Sympathetic and Parasympathetic Nervous System (SNS, PNS). Cholinergic nerve endings of the PNS, vagus nerve, slow the firing rate of the SA node - and, hence, the heart rate-, whereas noradrenergic sympathetic nerve endings have the opposite effect. The reciprocal effect of these arms of the ANS can be observed within an individual cardiac cycle. In addition, catecholamines released into the circulation by the adrenal glands-along with cortisol-further influence the rate and force of cardiac contraction. The regulation of vascular tone by SNS and PNS also contributes to cardiac performance: greater ventricular filling consequent to more rapid venous return will produce a stronger and more rapid ventricular contraction, because of the direct effects on the myocardial tissue, while an increase in peripheral vasoconstriction will also influence cardiac contraction as it works to move blood through the arterial system against the increased Total Peripheral Resistance (TPR). The CO depends both on the stroke volume - the volume of blood ejected by the ventricle during systole - and the heart rate-measured in

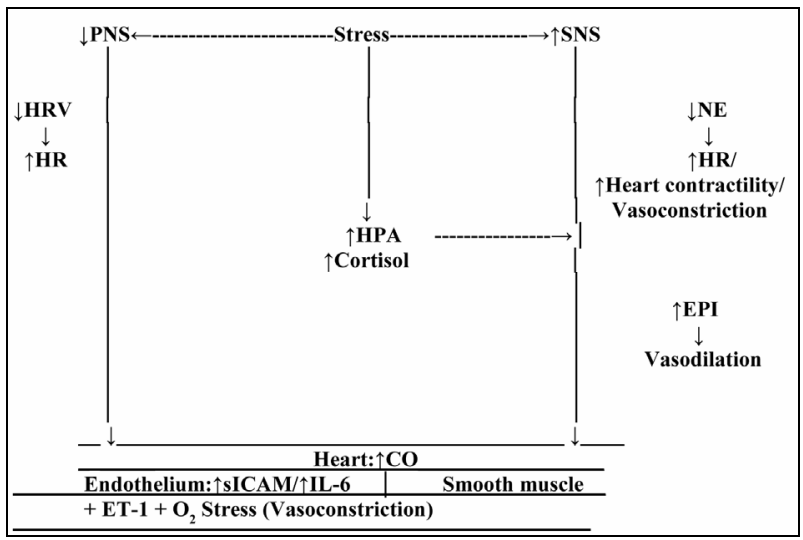

Figure 2. The regulation of Cardiac Output (CO) under conditions of stress. Stress results in activation of the two branches of the Autonomic Nervous System (ANS), and of the Hypothalamic-Pituitary-Adrenal (HPA) axis. Activation of the Sympathetic branch (SNS) results in PNS withdrawal, demonstrated by a reduction in High-Frequency (HF) Heart Rate Variability (HRV), and consequent increase in Heart Rate (HR). Activation of the Sympathetic branch (SNS) results in secretion of NorEpinephrine (NE) and Epinephrine (EPI), and consequent increase in both cardiac contractility and HR. Activation of the HPA axis is demonstrated by secretion of cortisol by the adrenal cortex, which both potentiates the effects of NE and EPI, and independently contributes to the same effects as these catecholamines. Respecting the regulation of smooth muscle tone under conditions of stress, activation of SNS results in Parasympathetic (PNS) withdrawal (reduction of HF-HRV). The consequent effects on the healthy endothelium are vasodilation, but, in the presence of atherosclerotic disease, vasoconstriction. PNS withdrawal leads to an increased release of pro-inflammatory cytokines - Interleulcin-6 (IL-6)- and vasoactive proteins-Intercellular Adhesion Molecule (sICAM) from macrophages, due to the resulting disinhibition. Activation of the SNS results in secretion NE and EPI, with consequent effects on vascular smooth muscle. Release of NE causes vasoconstriction, through its action on $\alpha$-receptors. EPI released into the circulation, from the adrenal medulla, provokes an opposite vasodilating effect, through its action on $\beta 1$-receptors. Activation of HPA axis, and subsequent secretion of cortisol, both potentiates the effects of NE and EPI, and, independently, contributes to the down-regulation of the inflammatory response, through its action on $1 \mathrm{~L}-6$, though in the presence of atherosclerotic disease, this negative feedback loop is impaired. Stress contributes to oxidative $\left(\mathrm{O}_{2}\right)$ stress, which contributes to endothelial dysfunction and consequent vasoconstriction, with Endothelin-1 (ET-I) playing an essential role (Adapted, with permission, from The Cardiovascular System, by Mathew, MB, Pickering, TG, in The Handbook of Stress Science: Psychology, Medicine and Health, edited by Contrada, RJ, Andrew, B, Springer Publishing Company, Inc. 2011).

beats per minute. The stroke volume is determined both by the amount of blood filling the heart during diastole (preload), and by the resistance to the heart's pumping (after-load). Essentially then, stroke volume depends on ventricular volumes at the end of the filling period-the end diastolic volume - and at the end of contraction — the 
end systolic volume. These volumes are determined by wide ranging factors including: venous return or preload, which contributes to the degree of stretch in the wall of the ventricle; because of the nature of myocardial tissue, an increase in venous return results in an increase in end diastolic volume and associated muscle stretch and, hence, an increase in stroke volume; and after-load, reflected in TPR. All of these factors will typically vary in an ongoing manner as determined by moment to moment changes in tissue metabolic requirements and ANS tone. The SNS stimulation can profoundly increase the Heart Rate (HR) (contractions per minute), the speed of individual ventricular contractions, and the force of these contractions (for example, shorter systolic and diastolic intervals, more forceful ejection, and faster acceleration of ventricular outflow velocities). Conversely, PNS stimulation can have immediate and profound effects, by slowing the heart rate. The vascular system - specifically its arterial branch-serves as a branching system that directs blood to each bodily organ according to its needs, and also serves as a "pressure reservoir" that absorbs the pulsations in blood flow produced by the alternating contraction and relaxation of the heart's ventricles. A reduction of arterial elasticity is one of the first alterations that can be observed along the path toward essential hypertension. Diastolic and systolic pressure can vary substantially as a function of metabolic needs and physical and emotional stress, though diastolic pressure is prevented by bodily reflexes from dropping below between 40 and $60 \mathrm{mmHg}$ so as to ensure a level of perfusion to the brain necessary to maintain consciousness. Cross-sectionally, arteries are described by a lumen, the open interior through which blood passes. A single layer of endothelial cells surrounds the lumen and this cellular layer is in turn surrounded by a thicker layer of smooth muscle cellsthese two layers provide avenues by which the ANS and other local processes dynamically regulate vascular tone. Smaller arteries and arterioles that are further along the arterial tree are notable for their greater muscularity, which allows them to serve as sphincters, that vasoconstrict or vasodilate, and thereby control the flow of blood to the capillary beds they serve, and it is through this involuntarily regulated function, blood flow to each bodily organ is precisely controlled according to factors that include relative local and systemic metabolic needs associated with levels and types of activity. Although perfusion to the organs can vary according to relative needs (increased blood flow to large muscles and heart can be associated with relative decrease in blood flow to digestive organs as observed during the "fight-flight" response), perfusion to the brain must be kept constant. Having passed through the capillary bed, the flow of blood in the venous return system is at a low pressure and moves proportionately slower (in contrast to arteries, therefore, veins have a larger lumen and thinner walls, so that resistance to flow is reduced). Internal one-way valves ensure that blood flows back to the heart, whereas contractions of muscles around the veins, such as those in the legs and abdomen, support the flow of blood against the force of gravity as it returns from the lower body and extremities back to the heart. Regulation of the circulation (Figure 2) is achieved by a combination of brain activity with local regulatory mechanisms: monitoring changes of oxygenation is accomplished by chemoreceptors, and arterial pressure, is accomplished by baroreceptors. Increased metabolic activity in muscles depletes oxygen and increases levels of carbon dioxide and associated chemical reactants, and chemoreceptors detect these changes and, in addition to signalling the brain that there is a need for increased respiration, provoke relative dilation in local microvascular smooth muscle to increase blood flow and thereby provide the oxygen needed to support the continued work of the muscle. Reactive hyperemia, an auto-regulatory process by which the vascular endothelium responds with significant nitric oxide provoked dilation after release of temporary occlusion of blood flow, provides an example of the underlying mechanism. Baroreceptors, with the most sensitive being found in carotid sinuses and aortic arch, comprise part of a system of Central Nervous System feedback loops that control ANS activity in the service of Blood Pressure (BP) regulation. SNS activation leads to an elevation of TPR and CO (via increased cardiac contractility, heart rate and arterial vasoconstriction, which together tends to increase BP). Conversely, PNS activation leads to a decreased CO (via decreased HR, resulting in a tendency to lower BP). SNS and/or PNS inhibition produces opposite results. When BP rises, the carotid and aortic sinuses are distended, resulting in stretch and therefore activation and firing of the baroreceptors. Alternatively, when BP drops, firing of baroreceptors decreases. Baroreceptor activation provokes inhibition of SNS activity and excitation of PNS activity. By coupling relative SNS inhibition and PNS activation, the baroreflex maximizes BP regulation. SNS inhibition leads to a drop in TPR, whereas PNS activation leads to depressed heart rate and myocardial contractility, which will dramatically decrease BP. Conversely, a decrease in BP is detected by baroreceptors, and, through these circuits, increases SNS activation, inhibits PNS activity and, through an increase in CO and TPR, elevates BP. The analysis of Heart Rate Variability (HRV) allows the observation of the fluctuations of HR during worship time periods or long periods and is a non-invasive and selective observation of the ANS function. These methods include simple statistics, such as the standard deviation 
of HR or RR interval. For analysis of cardio-vagal function, the methods developed for clinical trials typically involve the measurement of HRV over short intervals of time ( $<90$ seconds) $[41,42]$. To study the ANS under various conditions such as physiological, psychological and pathological, non-invasive analysis of spectral power in RR intervals has been used very recently [43]. The power spectrum is determined by measuring the area under the band of two frequencies: Low Frequency (LF) power, calculated from 0.04 to $0.15 \mathrm{~Hz}$, and High Frequency (HF), whose power ranges from 0.15 to $0.40 \mathrm{~Hz}$. The clinical usefulness of HRV to identify changes in the ANS, diseases of the heart, has been enhanced by the use of different methods of study. Time domain variability is measured by the statistical and geometric method, and the selection of each match the objective of each particular case. The study in the frequency domain is related to the analysis of the spectral density, which studies how power (variance) distributes as a function of frequency, i.e., spectral analysis decomposes the total HRV in their causing components, presenting them according to the frequency with which changes the HR. For the calculations of spectral density, are commonly used nonparametric (Fast Fourier Transform) and parametric (autoregressive model) methods, and these allow the reading of the same results, tough each have their own advantages. They decompose a signal in its frequency components, and, regardless of the calculation of the spectral density (non-parametric or parametric), it is usually delimited three distinct frequency bands, called spectral components [42,44], which are: the frequency band < $0.003 \mathrm{~Hz}$ (Ultra Low frequency: ULF) and the frequency band from 0.003 to $0.04 \mathrm{~Hz}$ (Very Low Frequency: VLF) represent actions of humoral regulation, vasomotor and temperature and the activity of renin-angiotensin-aldosterone system. The frequency band of 0.04 to $0.15 \mathrm{~Hz}$ (Low Frequency: LF) reflects the SNS and PNS modulation tone, through the baro-reflex activity (regulation of BP). The frequency band from 0.15 to 0.45 (High Frequency: HF) is the modulation of the vagal activity. With advancing age, HRV suffers a decline, i.e., varies less. In the normal population without pathological signs, HRV shows broad limits due to the adjustment of the ANS and age. Changes in posture, emotions, time of day or night can induce changes in this same variability [45]. With regard to gender, there are reports that middle-aged men present predominantly lower HRV when compared with women of similar age [46]. A reduced HRV is presented as a powerful predictor of arrhythmic complications resulting from a myocardial infarction and of mortality [42]. The weight also influences HR and HRV. Another factor that influences HRV has to do with exercise-exercise itself is a behavior that provokes important changes in the functioning of the cardiovascular system and its mechanisms of autonomic adjustments [47].

\subsection{Connectivity between Anterior Cingulate Cortex and Dorsolateral Prefrontal Cortex}

The anatomical and functional connections between DLPFC and medial prefrontal cortex, including Anterior Cingulated Cortex (ACC) and orbitofrontal cortex, have been documented by anatomical and neuroimaging studies [48-52]. ACC is the frontal part of the cingulate cortex-resembling a "collar" form around the corpus callosum, the fibrous bundle that relays neural signals between the right and left cerebral hemispheres of the brain - and it consists of Broadmann areas 24, 32 and 11, appearing to play a role in a wide variety of autonomic functions, such as regulating $\mathrm{BP}$ and $\mathrm{HR}$, as well as rational cognitive functions (reward anticipation, decision making, empathy, emotion). ACC can be divided anatomically based on cognitive (dorsal), and emotional (ventral) components - the dorsal part of the ACC is connected with the prefrontal cortex and parietal cortex as well as the motor system and the frontal eye fields, making it a central station for processing top-down and bottom-up stimuli and assigning appropriate control to other areas in the brain; the ventral part of the ACC is connected with amygdala, nucleus accumbens, hypothalamus, and interior insula, and is involved in assessing the salience of emotion and motivational information. The adjacent subcallosal cingulate gyrus has been conjectured to play an important role in major depression, and has been the target of deep brain stimulation to treat that disease [53]. Using indices of HRV, and HF and LF power in the cardiac rhythm, Critchley et al. (2003) observed activity in the dorsal ACC related to sympathetic modulation of HR that was dissociable from cognitive and motor-related activity, and their findings predict that during effortful cognitive and motor behaviour the dorsal ACC supports the generation of associated autonomic states of cardiovascular arousal (neuroimaging and clinical findings suggest that ACC function mediates context-driven modulation of bodily arousal states) [54].

\subsection{Summary of Transcranial Direct Current Stimulation and Heart Rate Variability}

Transcranial Direct Current Stimulation (tDCS) is a noninvasive, technique for brain stimulation. Anodal stimulation causes neuronal depolarisation and long-term potentiation, while cathodal stimulation causes hyperpolarisation and long-term depression. Stressors are associated with an increase in sympathetic cardiac control, a decrease in parasympathetic control, or both. Associated with these reactions is a frequently reported increase in LF HRV, a decrease in HF power, and/or an increase in 


\begin{tabular}{|l|l|l|l|r|}
\hline Category & \multicolumn{1}{|c|}{ Name } & \multicolumn{1}{c|}{ Measure } & \multicolumn{1}{c|}{ Unit } & Value \\
\hline \hline HRV & Spectral & TotPeak & $\mathrm{Hz}$ & 0.0025 \\
\hline HRV & Spectral & VLFPeak & $\mathrm{Hz}$ & 0.0166 \\
\hline HRV & Spectral & LFPeak & $\mathrm{Hz}$ & 0.0732 \\
\hline HRV & Spectral & HFPeak & $\mathrm{Hz}$ & 0.1623 \\
\hline HRV & Spectral & TotPower & $\mathrm{ms}^{\wedge} 2$ & 578.7 \\
\hline HRV & Spectral & VLFPower & $\mathrm{ms}^{\wedge} 2$ & 215.5 \\
\hline HRV & Spectral & LFPower & $\mathrm{ms}^{\wedge} 2$ & 217.9 \\
\hline HRV & Spectral & HFPower & $\mathrm{ms}^{\wedge} 2$ & 78.6 \\
\hline HRV & Spectral & LFPower & n.u. & 60 \\
\hline HRV & Spectral & HFPower & n.u. & 21.7 \\
\hline HRV & Spectral & LF/HF & ratio & 2.771 \\
\hline
\end{tabular}

\begin{tabular}{|l|l|l|l|r|}
\hline Category & \multicolumn{1}{|c|}{ Name } & \multicolumn{1}{c|}{ Measure } & \multicolumn{1}{c|}{ Unit } & Value \\
\hline \hline HRV & Spectral & TotPeak & $\mathrm{Hz}$ & 0.0066 \\
\hline HRV & Spectral & VLFPeak & $\mathrm{Hz}$ & 0.0066 \\
\hline HRV & Spectral & LFPeak & $\mathrm{Hz}$ & 0.0464 \\
\hline HRV & Spectral & HFPeak & $\mathrm{Hz}$ & 0.2185 \\
\hline HRV & Spectral & TotPower & $\mathrm{ms}^{\wedge} 2$ & 1363.7 \\
\hline HRV & Spectral & VLFPower & $\mathrm{ms}^{\wedge} 2$ & 652.9 \\
\hline HRV & Spectral & LFPower & $\mathrm{ms}^{\wedge} 2$ & 355.9 \\
\hline HRV & Spectral & HFPower & $\mathrm{ms}^{\wedge} 2$ & 327.4 \\
\hline HRV & Spectral & LFPower & n.u. & 50.1 \\
\hline HRV & Spectral & HFPower & n.u. & 46.1 \\
\hline HRV & Spectral & LF/HF & ratio & 1.087 \\
\hline
\end{tabular}

(3.4)

\begin{tabular}{|l|l|l|l|r|}
\hline Category & \multicolumn{1}{|c|}{ Name } & \multicolumn{1}{c|}{ Measure } & \multicolumn{1}{c|}{ Unit } & Value \\
\hline \hline HRV & Spectral & TotPeak & Hz & 0.0008 \\
\hline HRV & Spectral & VLFPeak & $\mathrm{Hz}$ & 0.0091 \\
\hline HRV & Spectral & LFPeak & $\mathrm{Hz}$ & 0.044 \\
\hline HRV & Spectral & HFPeak & $\mathrm{Hz}$ & 0.2208 \\
\hline HRV & Spectral & TotPower & $\mathrm{ms}^{\wedge} 2$ & 465.8 \\
\hline HRV & Spectral & VLFPower & $\mathrm{ms}^{\wedge} 2$ & 131 \\
\hline HRV & Spectral & LFPower & $\mathrm{ms}^{\wedge} 2$ & 176.2 \\
\hline HRV & Spectral & HFPower & $\mathrm{ms}^{\wedge} 2$ & 76.2 \\
\hline HRV & Spectral & LFPower & n.u. & 52.7 \\
\hline HRV & Spectral & HFPower & n.u. & 22.8 \\
\hline HRV & Spectral & LF/HF & ratio & 2.312 \\
\hline
\end{tabular}

\begin{tabular}{|l|l|l|l|r|}
\hline Category & \multicolumn{1}{|c|}{ Name } & \multicolumn{1}{c|}{ Measure } & \multicolumn{1}{c|}{ Unit } & Value \\
\hline \hline HRV & Spectral & TotPeak & $\mathrm{Hz}$ & 0.0022 \\
\hline HRV & Spectral & VLFPeak & $\mathrm{Hz}$ & 0.0059 \\
\hline HRV & Spectral & LFPeak & $\mathrm{Hz}$ & 0.0452 \\
\hline HRV & Spectral & HFPeak & $\mathrm{Hz}$ & 0.3076 \\
\hline HRV & Spectral & TotPower & $\mathrm{ms}^{\wedge} 2$ & 892.8 \\
\hline HRV & Spectral & VLFPower & $\mathrm{ms}^{\wedge} 2$ & 332.1 \\
\hline HRV & Spectral & LFPower & $\mathrm{ms}^{\wedge} 2$ & 187.6 \\
\hline HRV & Spectral & HFPower & $\mathrm{ms}^{\wedge} 2$ & 266.6 \\
\hline HRV & Spectral & LFPower & n.u. & 33.5 \\
\hline HRV & Spectral & HFPower & n.u. & 47.6 \\
\hline HRV & Spectral & LF/HF & ratio & 0.704 \\
\hline
\end{tabular}
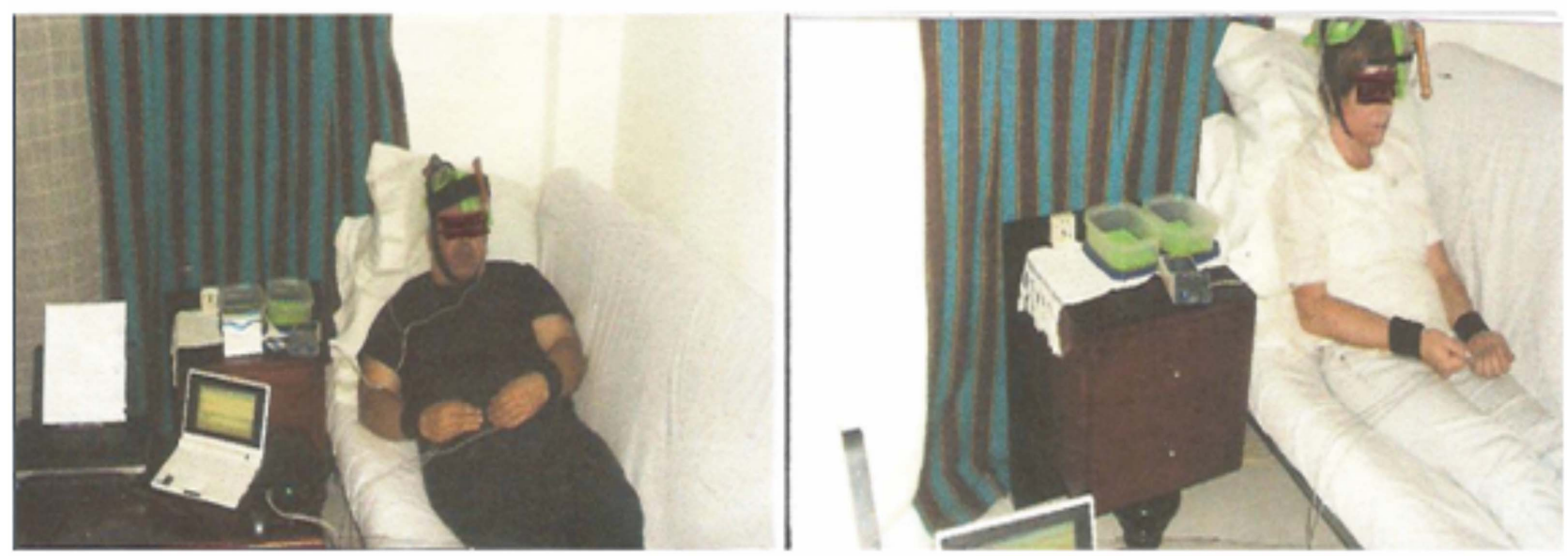

Figure 3. HRV parameters result (Cstress Analysis) - Total Peak, VLF Peak, LF Peak, HF Peak, Total Power, VLF Power, LF Power, HF Power and LF/HF ratio-before (3.1) and after (3.2) tDCS (using stimulater Phoresor II Auto model from Iomed Inc., USA) in the male subject, and before (3.3) and after (3.4) tDCS in the female subject. 
the $\mathrm{LF} / \mathrm{HF}$ ratio.

\subsection{Objectives and Aims}

The present work aims to explore the tDCS potential in the modulation of ANS, through indirect stimulation of ACC.

\subsection{Methods}

Two subjects, a 39 year old female and a 49 year old male, gave informed consent. Saline soaked synthetic sponges involving two, thick, metallic (stainless steel) rectangles, with an area of $25 \mathrm{~cm}^{2}$ each have been used as electrodes, connected to Iomed Phoresor II Auto device. It has been delivered a $2 \mathrm{~mA}$ current, for 20 minutes, over the left DLPFC (Anode). Spectrum analysis (cStress software) of HRV has been performed before and after tDCS administration.

\subsection{Results}

The female/male suject results of LF power, HF power and LF/HF ratio, before tDCS administration, were, respectively: $50.1 \mathrm{nu} / 60 \mathrm{nu}, 46.1 \mathrm{nu} / 21.7 \mathrm{nu}$ and 1.087/ 2.771; and, after tDCS administration, respectively: 33.5 $\mathrm{nu} / 52.7 \mathrm{nu}, 47.6 \mathrm{nu} / 22.8 \mathrm{nu}$ and 0.704/2.312 (Figure 3).

\subsection{Limitations}

The present study is limited by the quantity of research subjects; it is deprived of the blinding- and sham-control nature in its design.

\subsection{Conclusions}

tDCS over the left DLPFC (left ACC) increased parasympathetic activity and decreased sympathetic activity, suggesting the importance of tDCS in the management of stress-related disorders.

To our best knowledge, this is the first attempt to operationalize the concept of Neurostimulation Related Heart Rate (Frequency) (NRF)/tDCS Evoked Heart Rate (Frequency) (TEF).

\section{ACKNOWLEDGEMENTS}

The authors gratefully acknowledge the comments of Prof. Helen Mayberg (from Emory School of Medicine, USA).

\section{REFERENCES}

[1] Priori, A. (2003) Brain polarization in humans: A reappraisal of an old tool for prolonged non-invasive modulation of brain excitability. Clinical Neurophysiology, 114, 589-595. doi:10.1016/S1388-2457(02)00437-6

[2] Antal, A, Kincses, T.Z., Nitsche, M.A., Bartfai, O. and
Paulus, W. (2004) Excitability changes induced in the human primary visual cortex by transcranial direct current stimulation: Direct electrophysiological evidence. Investigative Ophthalmology \& Visual Science, 45, 702707. doi:10.1167/iovs.03-0688

[3] Antal, A., Nitsche, M.A. and Paulus, W. (2001) External modulation of visual perception in humans. Neuroreport, 12, 3553-3555. doi:10.1097/00001756-200111160-00036

[4] Antal, A., Nitsche, M.A., Kruse, W., et al. (2004) Direct current stimulation over V5 enhances visuo-motor coordination by improving motion perception in humans. Journal of Cognitive Neuroscience, 16, 521-527. doi:10.1162/089892904323057263

[5] Antal, A., Nitsche, M.A., Kincses, T.Z., et al. (2004) Facilitation of visuo-motor learning by transcranial direct current stimulation of the motor and extrastriate visual areas in humans. European Journal of Neuroscience, 19, 2888-2892. doi:10.1111/j.1460-9568.2004.03367.x

[6] Marshall, L., Mölle, M., Hallsehmid, M. and Born, J. (2004) Transcranial direct current stimulation during sleep improves declarative memory. Journal of Neuroscience, 24, 9985-9992.

doi:10.1523/JNEUROSCI.2725-04.2004

[7] Ragert, P., Vandermeeren, Y., Camus, M. and Cohen, L.G. (2008) Improvement of spatial tactile acuity by transcranial direct current stimulation. Clinical Neurophysiology, 119, 805-811. doi:10.1016/j.clinph.2007.12.001

[8] Monti, A., Cogiamanian, F., Marceglia, S., et al. (2008) Improved naming after trancranial direct current stimulation in aphasia. Journal of Neurology Neurosurgery \& Psychiatry, 79, 451-453. doi:10.1136/jnnp.2007.135277

[9] Ferrucci, R., Mameli, F., Guidi, I., et al. (2008) Recognition memory in Alzheimer disease. Neurology, 34, $267-$ 281.

[10] Flöel, A., Rosser, N., Michka, O., Knecht, S. and Breitenstein, C. (2008) Non-invasive brain stimulation improves language learning. Journal of Cognitive Neuroscience, 20, 1415-1422. doi:10.1162/jocn.2008.20098

[11] Sparing, R., Dafotakis, M. and Meister, I.G. Thirugnanasambandam, N. and Fink, G.R. (2008) Enhancing language performance with non-invasive brain stimulation-A transcranial direct current stimulation study in healthy humans. Neuropsychologia, 46, 261-268. doi:10.1016/j.neuropsychologia.2007.07.009

[12] Hummel, F.C., Celnik, P., Giraux, P., et al. (2005) Effects of non-invasive cortical stimulation on skilled motor function in chronic stroke. Brain, 128, 490-499. doi:10.1093/brain/awh369

[13] Nitsche, M.A., Schauenburg, A., Lang, N., et al. (2003) Facilitation of implicit motor learning by weak transeranial direct current stimulation of the primary motor cortex in the human. Journal of Cognitive Neuroscience, 15 619-626. doi:10.1162/089892903321662994

[14] Fregni, F., Boggio, P.S., Nitsche, M., et al. (2005) Anodal transcranial direct current stimulation of prefrontal cortex enhances working memory. Experimental Brain Research, 166, 23-30. doi:10.1007/s00221-005-2334-6

[15] Boggio, P.S., Ferrucci, R., Rigonatti, S.P., et al. (2006) 
Effects of transcranial direct current stimulation on working memory in patients with Parkinson's disease. Journal of the Neurological Science, 249, 31-38. doi:10.1016/j.jns.2006.05.062

[16] Ohn, S.H., Park, C.I., Yoo, W.K., et al. (2008) Timedependent effect of transcranial direct current stimulation on the enhancement of working memory. Neuroreport, 19, 43-47. doi:10.1097/WNR.0b013e3282f2adfd

[17] Roizenblatt, S., Fregni, F., Gimenez, R., et al. (2007) Site-specific effects or transcranial direct current stimulation on sleep and pain in fibromyalgia: A randomized, sharn-controlled study. Pain Practice, 7, 297-306. doi:10.1111/j.1533-2500.2007.00152.x

[18] Fregni, F., Boggio, P.S., Lima, M.C., et al. (2006) A sham-controlled, phase II trial of transcranial direct current stimulation for the treatment of central pain in traumatic spinal cord injury. Pain, 122, 197-209. doi:10.1016/j.pain.2006.02.023

[19] Antal, A., Brepohl, N., Poreisz, C., et al. (2008) Transcranial direct current stimulation over somatosensory cortex decreases experimentally induced acute pain perception. Clinical Journal of Pain, 24, 56-63. doi:10.1097/AJP.0b013e318157233b

[20] Antal, A., Lang, N., Boros, K., et al. (2008) Homeostatic metaplasticity of the motor cortex is altered during headache-free intervals in migraine with aura. Cereb Cortex, 18, 2701-2705. doi:10.1093/cercor/bhn032

[21] Chadaide, Z., Arlt, S., Antal, A., et al. (2007) Transcranial direct current stimulation reveals inhibitory deficiency in migraine. Cephalalgia, 27, 833-839. doi:10.1111/j.1468-2982.2007.01337.x

[22] Fregni, F., Girnenes, R., Valle, A.C., et al. (2006) A randomized, sham-controlled, proof of principle study of transcranial direct current stimulation for the treatment of pain in fibromyalgia. Arthritis \& Rheumatism, 54, 39883998. doi:10.1002/art.22195

[23] Fecteau, S., Pascual-Leone, A., Zald, D.H., et al. (2007) Activation of prefrontal cortex by transcranial direct current stimulation reduces appetite for risk during ambiguous decision making. Journal of Neuroscience, 27, 62126218. doi:10.1523/JNEUROSCI.0314-07.2007

[24] Huey, E.D., Probasco, J.C., Moll, J., et al. (2007) No effect of DC brain polarization on verbal fluency in patients with advanced frontotemporal dementia. Clinical Neurophysiology, 118, 1417-1418. doi:10.1016/i.clinph.2007.02.026

[25] Boggio, P.S., Sultani, N., Fecteau, S., et al. (2008) Prefrontal cortex modulation using transcranial DC stimulation reduces alcohol craving: A double-blind, sham-controlled study. Drug Alcohol Dependence, 92, 55-60. doi:10.1016/j.drugalcdep.2007.06.011

[26] Fecteau, S., Knoch, O., Fregni, F., et al. (2007) Diminishing risk-taking behavior by modulating activity in the prefrontal cortex: A direct current stimulation study. Journal of Neuroscience, 27, 12500-12505. doi:10.1523/JNEUROSCI.3283-07.2007

[27] Fregni, F., Boggio, P.S., Nitsche, M.A., et al. (2006) Treatment of major depression with transcranial direct current stimulation. Bipolar Disorder, 8, 203-204. doi:10.1111/j.1399-5618.2006.00291.x

[28] Costain, R., Redfearn, J.W. and Lippold, O.C. (1964) A controlled trial of the therapeutic effect of polarization of the brain in depressive illness. British Journal of Psychiatry, 2, 786-799. doi:10.1192/bjp.110.469.786

[29] Carney, M.W. (1969) Negative polarization of the brain in the treatment of manic states. Irish Journal of Medical Sciences, 8, 133-135. doi:10.1007/BF02958921

[30] Bindman, L.J., Lippold, O.C.J. and Redfearn, J.W.T. (1964) The action of brief polarizing currents on the cerebral cortex of the rat (1) during current flow and (2) in the production of long-lasting after-effects. Journal of Physiology, 172, 369-382.

[31] Purpura, D.P. and McMurtry, J.G. (1965) Intracellular activities and evoked potential changes during polarization of motor cortex. Journal of Neurophysiology, 28, 166-185.

[32] Nitsche, M.A. and Paulus, W. (2000) Excitability changes induced in the human motor cortex by weak transcranial direct current stimulation. Journal of Physiology, 527, 633-639. doi:10.1111/j.1469-7793.2000.t01-1-00633.x

[33] Iyer, M.B., Mattu, U., Grafrnan, J., et al. (2005) Safety and cognitive effect of frontal DC brain polarization in healthy individuals. Neurology, 64, 872-875. doi:10.1212/01.WNL.0000152986.07469.E9

[34] Nitsche, M.A. and Paulus, W. (2001) Sustained excitability elevations induced by transcranial DC motor cortex stimulation in humans. Neurology, 57, 1899-1901.

[35] Nitsche, M.A., Nitsche, M.S., Klein, C.C., et al. (2003) Level of action of cathodal DC polarization induced inhibition of the human motor cortex. Clinical Neurophysiology, 114, 600-604. doi:10.1016/S1388-2457(02)00412-1

[36] Priori, A., Berardelli, A., Rona, S., Accornero, N. and Manfredi, M. (1998) Polarization of the human motor cortex through the scalp. Neuroreport, 9, 2257-2260. doi:10.1097/00001756-199807130-00020

[37] Dundas, J.E., Thickbroom, G.W. and Mastaglia, F.L. (2007) Perception of cornfort during transcranial DC stimulation: Effect of $\mathrm{NaCl}$ solution concentration applied to sponge electrodes. Clinical Neurophysiology, 118, 1166-1170. doi:10.1016/j.clinph.2007.01.010

[38] Ardolino, G., Bossi, B., Barbieri, S. and Priori, A. (2005) Non-synaptic mechanisms underlie the after-effects of cathodal transcutaneous direct current stimulation of the human brain. Journal of Physiology, 568, 653-663. doi:10.1113/jphysiol.2005.088310

[39] Agnew, W.F. and McCreery, D.B. (1987) Considerations for safety in the use of extracranial stimulation for motor evoked potentials. Neurosurgery, 20, 143-147. doi:10.1097/00006123-198701000-00030

[40] Liebetanz, D., Klinker, F., Hering, D., et al. (2006) Anticonvulsant effects of transcranial direct current stimulation (tDCS) in the rat cortical ramp model of focal epilepsy. Epilepsia, 47, 1216-1224. doi:10.1111/j.1528-1167.2006.00539.x

[41] Malik, M. (1996) Heart rate variability. Circulation, 93, 1043-1065. doi:10.1161/01.CIR.93.5.1043 
[42] Roberts, W. (2009) Heart rate variability with deep breathing as a clinical test of cardiovagal function. Cleveland Clinic Journal of Medicine, 76, S37-S40. doi:10.3949/ccjm.76.s2.08

[43] Shin, K., Minamitani, H., Onishi, S., Yamazaki, H. and Lee, M. (1997) Autonomic differences between athletes and nonathletes: Spectral analysis approach. Medicine \& Science in Sports \& Exercise, 29, 1482-1490. doi:10.1097/00005768-199711000-00015

[44] Akselrod, S., Gordon, D., Ubel, A., Shannon, C., Barger, C. and Cohen, J. (1981) Power spectrum analysis of heart rate fluctuation: A quantitative probe of beat-to-beat cardiovascular control. American Association for the Advancement of Science, 213, 220-222.

[45] Migliaro, R., Etxagibel, A., Castro, R., Ricca, R. and Vicente, K. (2001) Relative influence of age and sedentary life style in short-term analysis of heart rate variability. Brazilian Journal of Medical and Biological Research, 34, 493-500. doi:10.1590/S0100-879X2001000400009

[46] Bigger, J., Steinman, R., Rolnitzky, L., Fleiss, J., Albrecht, P. and Cohen, R. (1996) Power law behavior of RR-interval variability in healthy middle-aged persons, patients with recent acute myocardial infarction, and patients with heart transplants. Calculation, 93, 2142-2151.

[47] Mathew, M.B. and Pickering, T.G. (2011) The cardiovascular system, in the handbook of stress science: Psychology, medicine and health. Springer Publishing Company, Inc., New York, 37-45.

[48] Vogt, B.A. and Pandya, D.N. (1987) Cingulate cortex of the rhesus monkey: II. Cortical afferents. The Journal of Comparative Neurology, 262, 271-289.

doi:10.1002/cne.902620208

[49] Petrides, M. and Pandya, D.N. (1999) Dorsolateral prefrontal cortex: Comparative cytoarchitectonic analysis in the human and the macaque brain and cortico-cortical connection patterns. European Journal of Neuroscience, 11, 1011-1036. doi:10.1046/j.1460-9568.1999.00518.x

[50] Koski, L. and Paus, T. (2000) Functional connectivity of the anterior cingulate cortex within the human frontal lobe: A brain-mapping meta-analysis. Experimental Brain Research, 133, 55-65. doi:10.1007/s002210000400

[51] Paus, T., Castro-Alamancos, M.A. and Petrides, M. (2001) Cortico-cortical connectivity of the human mid-dorsolateral frontal cortex and its modulation by repetitive transcranial magnetic stimulation. European Journal of Neuroscience, 14, 1405-1411. doi:10.1046/j.0953-816x.2001.01757.x

[52] Mayberg, H.S., Lozano, A.M., Voon, V., McNeely, H.E., Seminowicz, D., et al. (2005) Deep brain stimulation for treatment-resistant depression. Neuron, 45, 651-660. doi:10.1016/j.neuron.2005.02.014

[53] Hamani, C., Mayberg, H.S., Stone, S., Laxton, A., Haber, S. and Lozano, A.M. (2001) The subcallosal cingulate gyrus in the context ofmajor depression. Biological Psychiatry, 69, 301-309. doi:10.1016/j.biopsych.2010.09.034

[54] Critchley, H.D., Mathias, C.J., Josephs, O., O’Doherty, J., et al. (2003) Human cingulate cortex and autonomic control: Converging neuroimaging and clinical evidence. Brain, 126, 2139-2152. doi:10.1093/brain/awg216 